\title{
H.I.I.T. HIGH INTENSITY IDEATION TRAINING - FACILITATING GROUP DESIGN IDEATION IN A DIGITAL TEACHING SPACE
}

\author{
Max John POWNALL, Emily Elizabeth BROOK and Kathryn PASHLEY \\ Nottingham Trent University
}

\begin{abstract}
2020/21 has been a challenging experience across Higher Education. Within the Product Design Department at NTU, these challenges have created opportunities to expand teaching methods and explore innovative alternatives. Utilizing these challenges to adapt to an online environment this paper provides an early adopter route into digital ideation training, whilst providing a clear roadmap to a future Blended Learning approach. Collaborative ideation is key to facilitating peer learning amongst 1st year design students, allowing them to build key Designer skills whilst fostering an environment of inclusivity and creativity. This is a recurrent challenge when transitioning to Higher Education, with the effect of COVID-19 only amplifying this. In 2021, many 1st year students had been out of education for 6 months before beginning University, rendering the challenge even greater. In response to the challenge, this paper proposes a new approach to collaboration, actively using the flexibility of online workspaces to create a new ideation paradigm.

Using the fundamental principles of Lotus Blossom Ideation approximately 100 students participated in High Intensity Ideation Training (HIIT) using Miro, an Online Visual Collaboration Platform.

This paper postulates that implementing this method online is not only highly successful but presents potential for more collaborative and creative rapid ideation. The method provides students with a range of creative tools, enhancing strength of communication, whilst removing some existing barriers to learning. Recommendations can be made for improved implementation of sessions and the paper provides and outline template for anyone wishing to adopt the process.
\end{abstract}

Keywords: Ideation, online, pedagogy, collaboration, HIIT

\section{CONTEXT}

The 2021/21 Academic year has brought challenges to education and design that have never been experienced before. Hands on, practical courses have been adapted to $100 \%$ online learning, and the issue of engagement and student success has never been more difficult to manage. It has not been without its opportunities however, as the rapid move to online teaching has brought to light innovative solutions to age old problems. One of these opportunities is the potential for the integration of Blended Learning to Product Design degrees. The mixed media approach provides potential to integrate with a wider group of learners and develop rich online content. One area that has had difficulty transitioning however is the application of group ideation sessions within an online teaching environment. Is it possible to foster creativity and inclusion when Design students are spread across the globe? Can students be taught to build on each other designs and actively participate without using paper and pens? The authors believe this is possible, and this paper proposes a solution to the challenge. Here we provide an early adopter route into online digital ideation methods and considering whether this is purely an emergency remote teaching method [2] or whether there is scope for a meaningful alternative to in person group studio ideation.

\section{METHODOLOGY}

Using the fundamental principles of Lotus Blossom Ideation [1] approximately 100 students participated in High Intensity Ideation Training (HIIT) using an Online Visual Collaboration Platform (Miro). Divided into small virtual groups, students completed a warm-up task, before moving into larger groups to build upon ideas and collaborate with peers. The Design Thinking method of 'How Might We' 
(HMW)[3] positional questions was used to drive the group ideation in a targeted direction. The intention was to see if it was possible to create new digital interactions by using existing in-person techniques in an innovative way, hoping that these new innovations can then be applied in a more blended approach once teaching in person returns.

\subsection{Framework}

An outline framework of the process used is described in Table 1 below. The process outlined was employed for several reasons. Drawing from existing literature around online interaction we found that 'when designing tasks, instructors should make assignments challenging, varied, active, multidimensional, and relevant. During the course, instructors should take steps to minimize students' anxiety, use effective evaluation and feedback, and promote interaction.' [4]. The framework was developed with these specific criteria considered to ensure students remained motivated throughout the process.

Table 1. HIIT Online Session Framework

\begin{tabular}{|c|c|c|}
\hline Stage & Description & Duration \\
\hline Set Up & $\begin{array}{l}\text { Single online meeting utilizing chat function. This allows single Tutor to } \\
\text { control session overall \& issue instructions, with support Tutors divided } \\
\text { across group Miro boards. }\end{array}$ & \\
\hline $\begin{array}{c}30 \\
\text { Apples }\end{array}$ & $\begin{array}{l}\text { Using pre created Miro boards the groups begin to ideate around the } \\
\text { concept of } 30 \text { different apples. Allow familiarization with Miro \& tools }\end{array}$ & $\begin{array}{c}10 \\
\text { minutes }\end{array}$ \\
\hline $\begin{array}{l}\text { HMW } \\
\text { Themes }\end{array}$ & $\begin{array}{l}5 \text { statements spread across groups, with } 4 \text { groups ( } 25 \text { students per group) } \\
\text { working on the same statement. Ensure briefs are not related to current } \\
\text { project. Example themes- How might we carry water? How might we light a } \\
\text { room? Etc. Task is to think of } 8 \text { themes within HMW statements as a group. } \\
\text { An unrelated example is provided to demonstrate concept. } \\
\text { Each Miro board has the brief in the middle to allow group to surround it } \\
\text { with themes. This board is utilized for ideas in the next two exercises. }\end{array}$ & $\begin{array}{l}10 \\
\text { minutes }\end{array}$ \\
\hline \multirow[t]{2}{*}{$\begin{array}{c}\text { Idea } \\
\text { Rotation }\end{array}$} & $\begin{array}{l}\text { Each group member picks a theme and has } 5 \text { minutes to draw as many } \\
\text { ideas as possible on the Miro board. After } 5 \text { min move to the next theme } \\
\text { and rotate until all themes are complete. Encourage drawing and being } \\
\text { loose with ideas. Ideally } 40 \text { ideas per theme are generated. }\end{array}$ & $\begin{array}{c}45 \\
\text { minutes }\end{array}$ \\
\hline & BREAK & \\
\hline \multirow[t]{2}{*}{$\begin{array}{l}\text { Cluster } \\
\text { Rumble }\end{array}$} & $\begin{array}{l}\text { As a group, the students cluster their ideas within each theme highlighting } \\
\text { shared characteristics. Encourage all to participate in discussion. }\end{array}$ & $\begin{array}{c}30 \\
\text { minutes }\end{array}$ \\
\hline & BREAK & \\
\hline $\begin{array}{c}\text { Decision } \\
\text { Point }\end{array}$ & $\begin{array}{l}\text { Groups select } 8 \text { ideas they think have the potential to be explored further. } \\
\text { Ideas developed into more defined concepts and shared across cohort. }\end{array}$ & $\begin{array}{c}15 \\
\text { minutes }\end{array}$ \\
\hline $\begin{array}{l}\text { Cool } \\
\text { Down }\end{array}$ & $\begin{array}{l}\text { Presentation of concepts for each brief, tutor and student comments and } \\
\text { reflection. Participants highlight areas of interest. }\end{array}$ & $\begin{array}{c}15 \\
\text { minutes }\end{array}$ \\
\hline
\end{tabular}

\subsection{Apples}

The 30 apples ideation process was employed for two reasons. Firstly, as stated by the Creators 'The purpose of this simple exercise is to demonstrate three key principles useful for creativity and idea generation: quantity is a condition for quality; building on the ideas of others; the ideas we come up with are usually all the same.' [5]. Secondly, the process acts as an ice breaking activity, allowing students to not only become accustomed with Miro and its tools, but also to begin to 'lose the ego' surround drawing quality. All participant skills are limited to the basic tools provided by Miro, therefore drawing quality is less of a factor.

\subsection{HIIT}

The HIIT session itself allows the students to build on the outlined themes individually whilst working as part of a larger team. This concept of 'alone-together' helps to promote a feeling of belonging within the virtual group, without the need for verbal communication. 
The outlined themes are driven from the HMW questioning method using How Might We statements to "explore further aspects of a given problem, so that there is an appropriate problem for the subsequent Design Thinking processes.."[3] By framing the initial question as a HMW statement, students can ideate divergently within a theme, whilst not being restricted by it. This ensures everything that is created is meaningful and on task.

Building upon the ideas of the Lotus Blossom Ideation Method [1] the HIIT session allows ideas to be displayed visually, whilst remaining connected to the core theme. The process and layout of Lotus quickly highlights the quality of ideas (based on the number of concepts generated within that theme) and allows for rapid group decision making on which themes hold the most promise, or where more focus needs to be spent. The visual nature of the process also allows a visual narrative of ideas to form, showing the inception of an idea, and how the group have worked to iterate around that initial design.

Developing on from these principles, HIIT employs a further area of idea development using a 'Cluster Rumble.' Here ideas are grouped together to form ideas with shared characteristics that have potential for development. As a group within the Cluster Rumble, the students take these grouped ideas and begin to form more solid concepts from them. These are eventually filtered down to a select 8 which become the key concepts from the ideation activity.

The HIIT method of ideation is built around the idea of high intensity interval training in exercise. The short bursts of high intensity workouts have been shown to improve fitness, and the same can also be said for ideation. Drawing upon other task management styles such as Pomodoro [6] HIIT breaks down sections into rapid areas of activity, followed by breaks. This framework is outlined in Figure 1 below.

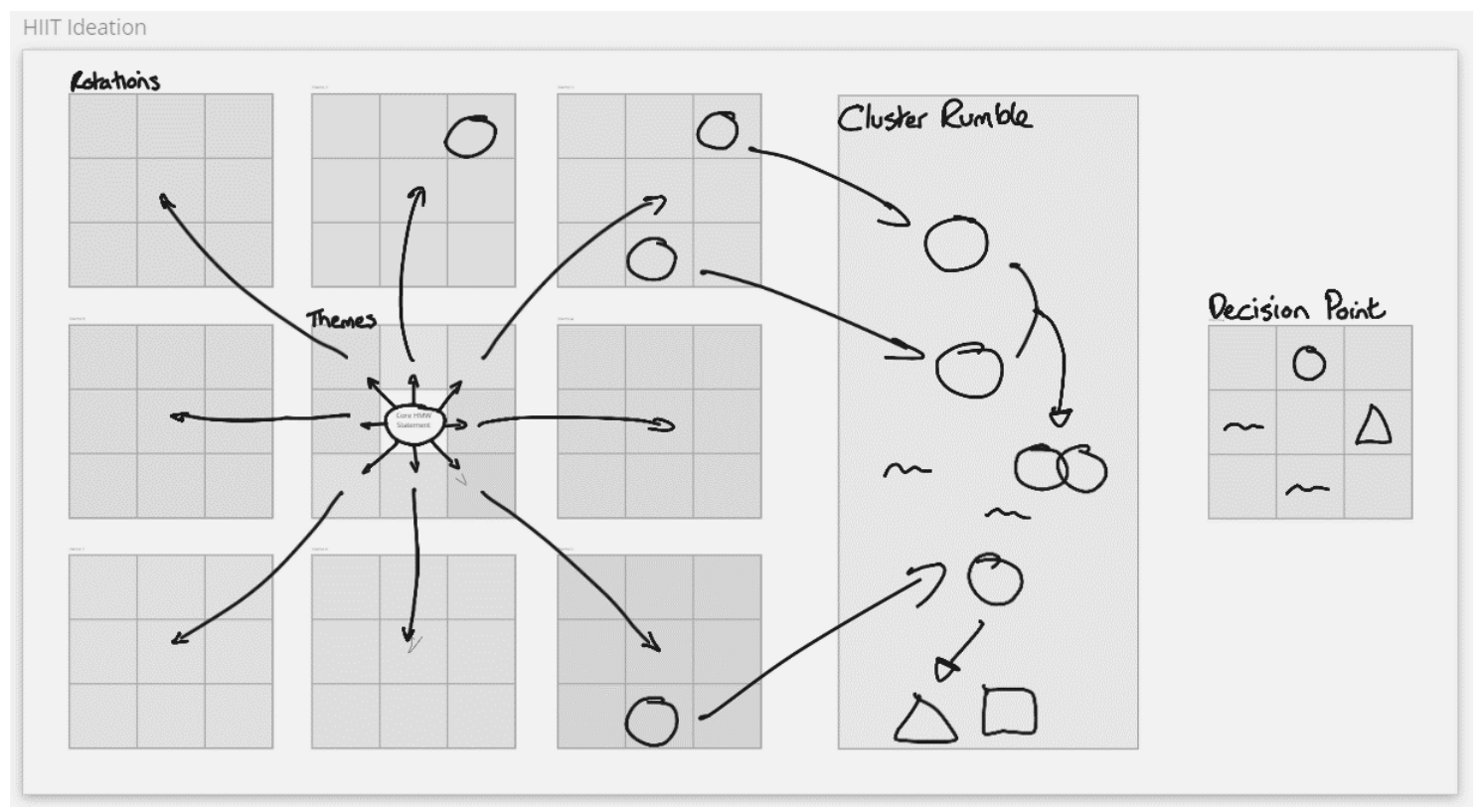

Figure 1. HIIT Framework

\section{FINDINGS}

\subsection{HIIT}

The High Intensity Ideation Training format was successful, with students engaging with the process to a greater degree than previously seen in on-site teaching. This paper postulates that implementing this method using an online setting is not only possible but presents potential for more collaborative and creative rapid ideation. Utilizing HIIT provides students with a range of creative tools, enhancing and developing strength of communication using colour, text, and reference images. Additionally, the use of Miro levels out ability by encouraging sketching through a computer mouse or trackpad, giving students with lower ability more confidence. Most promising is that students were observed to build upon each other's ideas, giving feedback, collaborating, and developing concepts as teams. HIIT helps to develop active, participatory student-centred learning environments that promotes instructor-student collaborations. [7] Existing barriers to learning still exist in this space, with a lower uptake from less 
engaged students and those who lack confidence. There is also a challenge in ensuring more confident students do not dominate the space, allowing students to contribute equally.

\subsection{Apples}

The use of 30 Apples as a warmup activity was highly successful, as it encourages participation without worry of failure, and ensured the participants were comfortable with the tools before commencing the activity. (Figure 2)

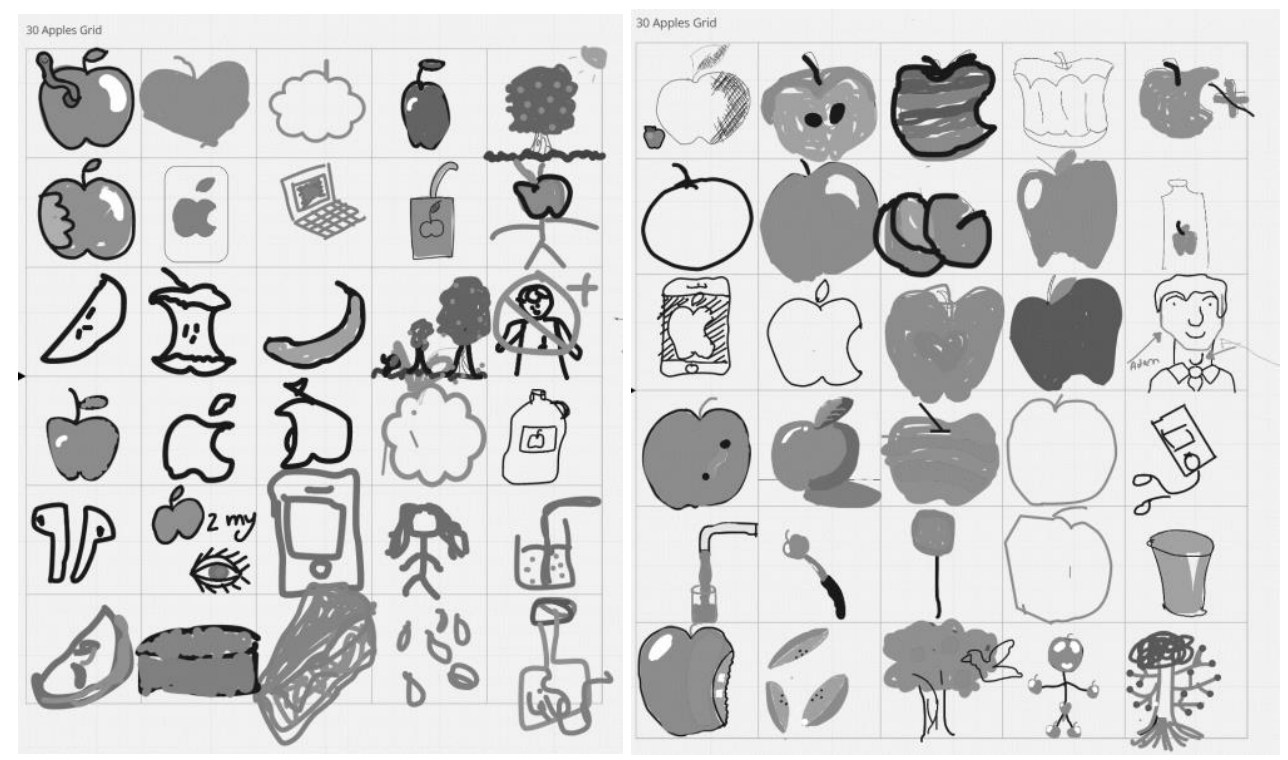

Figure 2. Warm up activity example

30 Apples also provides another function, as the tools within Miro can become a distraction and students can easily derail sessions if not closely monitored. Rapid ideation and collaboration are integral to the HIIT framework, but if not closely monitored they can result in off topic creativity. By providing a space for this creativity, HIIT sessions can be run more effectively by encouraging rapid ideation and collaboration without disruption. Careful considerations and monitoring by Tutors are needed within the task to ensure students do not become side-tracked.

\subsection{Cluster Rumble}

Cluster Rumble instils a feeling of shared ownership within the concepts, where students build on each other decisions to develop a concept greater than the sum of its parts. It also provides a granularity to the process, allowing iteration within ideas (a key skill often lacking in first year Design students,) rather than ideating with a fully formed concept from inception. The shared ownership of designs ensures students do not feel protective of their initial idea, as others have built upon it without the knowledge of the fully formed concept it was devised from.

\subsection{Anonymity}

Miro can be used anonymously, and most students opt for this posing both challenges and opportunities within HIIT workshops.

The positive impacts of anonymity within HIIT include increased confidence and willingness for collaboration. In remaining anonymous, it was observed that students are much quicker to collaborate with each other from the outset of the activity compared to in-person sessions. East[8] reports that anonymity gives students confidence to contribute, stating that participation "without the belief that they'd be identified as asking a stupid question, but 'someone' asking a stupid question." In building self-esteem among student cohorts, HIIT ideation sessions can remain short and sharp, with more ideas shared. East's [8] students reported that anonymity levelled the playing field amongst cohorts, and where students were not confident speaking within in-person sessions they were able to voice their opinions, questions, and thoughts through these online platforms more easily. In HIIT workshops, it was observed that anonymity provided a more level playing field, encouraging less confident students to participate in collaborative activity. 
As expected, one limitation of anonymous participation is that less engaged or less confident students can avoid participation. However, it should be noted that had the HIIT session been in person these students would have missed out completely due to non-attendance. With the HIIT sessions being online these students are still able to observe sessions even if they choose not to actively engage, therefore suggesting that the reach of HIIT online has a wider positive impact on students.

\subsection{Shared Ownership}

The aim of HIIT is rapid ideation, giving students a breadth of ideas to build from. When applying the Lotus Blossom Method to in-person sessions, it is observed that students often work individually on ideas before sharing with a wider group. This can be attributed to two key factors; students wanting to take individual ownership of ideas, or students lacking confidence in their own sketching ability, spending more time on the quality of the sketch itself rather than the quality of the idea. This often leads to a disconnect within a group and the collaborative element of Lotus Blossom is lost.

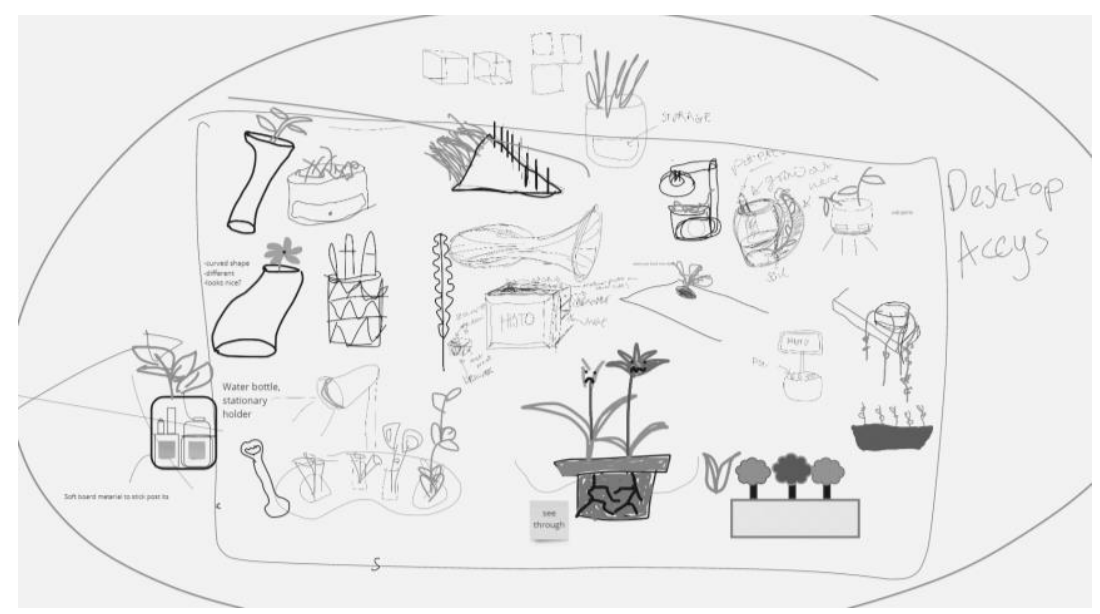

Figure 3. Example of Student Idea Collaboration

Miro helps to improve on this in several ways. Firstly, it allows all participants to see the actions of one another in real time, meaning multiple participants can work on the same idea synchronously. This early collaboration within a session encourages shared ownership of ideas from the outset (Figure 3). Furthermore, the tools within Miro level the playing field in terms of sketch ability, with most students sketching ideas using a computer mouse. Whilst the depth of detail that can be achieved with a mouse may be challenging, the basic function means that sketch ability plays less of a role in the generation of ideas. This allows students to ideate in a simple, less precise way and therefore builds confidence in those that are less comfortable with sketching. This leads to more rapid ideation and allows HIIT sessions to run in short creative bursts, allowing students to be more collaborative and take shared ownership of ideas.

\subsection{Tools for Creativity}

The tools available within Miro allow for more creativity within HIIT sessions. Typically, students come to in-person sessions with one pen or pencil, relying heavily on sketch ability to communicate ideas. Miro provides a variety of tools to better facilitate ideation. A range of pen colours, line thicknesses, shapes, and stickers aid in the communication of ideas by allowing students to give context without relying on a variety of physical equipment. In using the tools that Miro offers, students can express ideas creatively, allowing for quick collaboration and exploration of concepts. Furthermore, ideas can be better contextualised using existing images and media, which can be quickly copied into Miro. This adds a potential for greater depth of creativity as inspiration and reference points are integrated within ideation. Integration allows others to build upon concepts with a shared understanding of their origin.

\subsection{Measuring Success}

The success of HIIT can be measured through observation of student work in comparison to previous cohorts, with students that participated in HIIT having an increased ability in rapid ideation. This improved ideation ability is demonstrated through later projects and assessed work, with students 
producing a greater range of ideas compared to that previously expected of students from an A-Level background.

\section{CONCLUSIONS}

Overall, the experience of applying HIIT to an online learning environment was a positive one, with student collaboration and rapid ideation skills both being put into practise. A combination of anonymity and a level playing field in sketch ability allowed for enhanced confidence amongst the cohort, encouraging collaborative work and allowing students to build on and rapidly share ideas within the workspace. Furthermore, the availability of tools within Miro allows students to express ideas creatively, leading to a positive experience and giving students an opportunity to take shared ownership of work.

For optimum future implementation it is proposed that HIIT sessions be run as a blended learning experience, where students engage in an online collaborative space whilst in the same real-world environment. This would allow for shared benefits of digital collaboration across a cohort whilst promoting face to face interaction amongst peer groups. With a tutor driving the direction of HIIT, small student groups can collaborate in a real studio setting by contributing to a Miro board on a large screen, with participants highlighting key areas of innovation in real time. In doing so, students will benefit from the digitally collaborative aspects of Miro whilst building a real-world community.

Overall, the Authors found the application of HIIT sessions highly beneficial to students' creativity and learning, proving that collaboration amongst design students is possible even when working remotely. The hope is that with ever decreasing pandemic restrictions HIIT sessions develop and grow to become highly successful ideation sessions that utilise the benefits of online teaching, without being restricted by it.

\section{RECOMMENDATIONS}

Recommendations can always be made for implementing sessions and improving on their quality. The initial planning and setup of HIIT was time consuming. To reduce this initial set up time, we suggest utilising the Miro template provided in Figure 1 (available on request,) and for facilitators to follow the workshop framework outlined in Table 1. In following these guidelines, sessions should run smoothly with all staff having clarity on process and desired outcome, thus allowing more time for collaboration and on task learning. HIIT sessions are more successful with larger student groups of mixed ability and confidence, promoting collaboration and fostering a free-flowing environment.

Naturally, consideration needs to be given to technical difficulties that result from all versions of online teaching. During HIIT sessions, this can be tackled through splitting the cohort across Miro boards rather than aiming to have all students on one board. Whilst it is acknowledged that the session does work better with larger groups, technical limitations need to be taken into consideration to ensure the smooth running of the sessions.

\section{REFERENCE}

[1] Higgins J. M. 1996. Innovate or evaporate: creative techniques for strategists. Long Range Planning, 29 (3), 370-380.

[2] Hodges C. et al. 2020. The difference between emergency remote teaching and online learning. Educause Review, 27.

[3] Siemon D., Becker F., and Robra-Bissantz S. 2018. How might we? From design challenges to business innovation. Journal of Creativity and Business Innovation, 4.

[4] Beffa-Negrini P. A., Cohen N. L., and Miller B. 2002. Strategies to Motivate Students in Online Learning Environments. Journal of Nutrition Education and Behaviour, 34 (6), 334-340.

[5] Johannsson H., Reibke T., and Juhl Gregerson S. 2014. Apple Drawing- Ideation [online]. Available at: https://toolbox.hyperisland.com/apple-drawing-ideation-exercise [Accessed 26/01/ 2021].

[6] Cirillo F. 2006. The pomodoro technique (the pomodoro). Agile Processes in Software Engineering And, 54 (2), 35.

[7] Ouyang F. et al. 2020. Examining the instructor-student collaborative partnership in an online learning community course. Instructional Science, 1-22.

[8] East M. 2019. Giving students a voice: The power of anonymity in a digital learning environment [online]. Available at: https://talis.com/2019/07/25/giving-students-a-voice-the-power-ofanonymity-in-a-digital-learning-environment/. 